\title{
Constraining Minimum-Norm Inverse by Phase Synchronization and Signal Power of the Scalp EEG Channels
}

\author{
Kaushik Majumdar, Member, IEEE
}

\begin{abstract}
In this paper, the goal is to further improve the output of the scalp EEG source localization by the Euclidean minimumnorm (MN) inverse during single trials. Trials have been selected based on signal power at specific time intervals in specific locations. Then the source localization has been performed by MN. It has been observed that close to a dominant cortical source of EEG, as determined by the $\mathrm{MN}$, both pairwise phase synchronization of a channel with its nearest neighbors and the cumulative signal power of the channels within that neighborhood become high (normalized values remain above certain thresholds). This has also been verified through simulations on the subject's real head model. The conclusion of our study is that only those sources are to be chosen for which MN inverse, and signal power and phase synchronization profile converge. A novel fast Fourier transform (FFT) based phase synchronization measuring algorithm between a pair of signals has been developed whose time complexity is no more than that of the FFT.
\end{abstract}

Index Terms-Distributed source model, EEG, fast Fourier transform (FFT), minimum norm (MN), phase synchronization, real head model.

\section{INTRODUCTION}

I NTERPRETATION of the clinical EEG almost always involves speculation as to the possible locations of the sources inside the brain that are responsible for the observed activity on the scalp [1]. Simultaneous EEG-functional MRI (fMRI) recordings enable the investigation of trial-by-trial fluctuations of brain activity that reveals important insights into the dynamics of cognitive functions [2]. For this, localization of sources of the single-trial EEG signals and their comparison with the fMRI activities will be of great interest, because these two important brain signals apparently do not have positive correlations in many cases [2], [3]. Single-trial source localization may also give us insights about the transient nature of complex interactions among various brain regions [4]. The dominant trend in the EEG source localization has so far remained confined to estimating sources from the signals averaged over multiple trials in order to augment the signal-to-noise ratio (SNR) [5]-[7].

Manuscript received June 17, 2008; revised October 13, 2008. First published November 7, 2008; current version published May 6, 2009. This work was supported in part by the European Aeronautic Defence and Space Company (EADS) under Contract 2118.

The author was with the Odyssee Group, Institut National de Recherche en Informatique et en Automatique (INRIA) Sophia Antipolis, Sophia Antipolis 06902, France. He is now with the Center for Complex Systems and Brain Sciences, Florida Atlantic University, Boca Raton, FL 33431 USA (e-mail: mkkaushik@hotmail.com).

Color versions of one or more of the figures in this paper are available online at http://ieeexplore.ieee.org.

Digital Object Identifier 10.1109/TBME.2008.2008637
Single-trial EEG/magnetoencephalogram (MEG) source localization based on linear discrimination has been proposed in [7]. Single-trial source localization by means of expectation maximization algorithm based on Bayesian statistics has been proposed in [8]-[10]. In the former case, linear discriminators worked well for binary data, and in the latter case, the best result was obtained with accurate priors and Bayesian statistics based on a number of previous trials.

The study of synchronization in cortical activities has a long history [11]-[15]. Even controlling brain-computer interfaces using phase synchronization has been proposed [16]. Detection of synchronization is important in the study of epileptic seizures [17], [18] and Parkinson's disease [19], but unfortunately, there is no general agreement about the notion of synchronization in the signal processing community [20], biology, or even physics [21]. Various notions of phase synchronization and their comparative studies on EEG signals have been reported in [22]. The importance of the methods for understanding the complex interactions among brain regions using single-trial signals to investigate phase synchronization between pairwise sensors or directly within the MEG/EEG source map has been well recognized [4].

The purpose of our study is to improve the accuracy of singletrial source localization of the scalp EEG signals by simultaneous but independent application of two different techniques namely: 1) minimum-norm (MN) inverse and 2) phase synchronization and signal power profile of the scalp EEG channels. In this paper, we have run both the methods on the same dataset and only accepted those outputs in which they both agree. To improve the localization accuracy of the sources in single trials, selection of trials have been made based on strength of signals in the somatosensory area at the epoch determined by the EEGLAB [23] and supported by previous findings [24], [25]. The Euclidean $\mathrm{MN}\left(L_{2}\right.$-norm) and its various variations have so far remained the most widely used regularization techniques for the source localization inverse calculation [1], [4], [7]-[10]. In this paper, for single-trial source localization, we have followed the Euclidean $\mathrm{MN}$ regularization in a distributed source model setup [26]. A novel fast Fourier transform (FFT) based phase synchronization measuring algorithm between a pair of signals has been developed here.

In the next section, we will outline the methods used in this paper. In Section III, we will describe source localization on simulated EEG in the subject's real head model. Section IV contains data acquisition and preprocessing. In Section V, we will be presenting the results of source localization by simultaneous 
application of MN inverse, and signal power and phase synchronization profile on the EEG signal of the subject collected during median nerve stimulation. In the concluding section, we will summarize the results with a view to future directions.

\section{MEthODS}

\section{A. Trial Selection}

The scalp EEG signals collected from the subjects during stimulus presentation often contain hundreds of trials. Even after preprocessing, the signal strength across all the channels varies significantly from one trial to another. This variation is quite random across all the trials. Since the focus of our study is source localization in the single-trial EEG signals, selection of appropriate trials has been an important step. If the scalp EEG signal strength across all the channels in a trial is sufficiently weak, the cortical activity localization is likely to be faint and unreliable. To ensure adequate signal strength in the trial under experimentation, we have used the following straightforward algorithm.

\section{Proc(trial_select)}

1. Identify the time interval;

2. Identify the channels (default all channels);

3. Select trials serially \{

3.a) During the trial sum the square of the values of the discrete signal across the time interval at each selected channel;

3.b) Take the mean of the values obtained in 3.a) across all the selected channels. \}

4. Sort the values obtained in 3 in decreasing order across all the trials.

5. Select as many trials as required from the top (high valued);

It is important to note that once a trial is selected based on signal strength in a subset of channels, the actual source localization is done in that trial for the EEG signals from all the scalp channels.

\section{B. Forward and Inverse Calculations}

The head has been modeled as a set of nested regions (brain, skull, and scalp) with constant conductivities and separated by interfaces in between two mutually adjacent regions [22]. Each class of tissues of the head, like brain, skull, scalp, has been assumed to have constant conductivity ( 0 for air, 1 for scalp and brain, and 0.01 for skull). Based on these assumptions, the mathematical detail of the boundary-element-method-based forward calculation has been described in [27], which has also been schematically summarized in [28]. The sources of the scalp EEG have been identified with the Euclidean MN inverse calculation. The details of the inverse calculation can be found in [26] (also see [10]). Briefly, the following expression needs to be minimized:

$$
J(s)=\|H s-m\|^{2}+\lambda\|s\|^{2}
$$

where $s$ denotes the cortical source vector and $m$ denotes the scalp channel vector. If there are $p$ sources and $q$ channels, then $H$ is a $q \times p$ matrix, called the gain matrix, which is determined by the forward calculation. The parameter $\lambda$ is a regularization term (value taken as $10^{-20}$ in our calculations) introduced to make the ill-posed inverse problem a well-posed one. The righthand side of (1) has a unique minimum, and therefore, the minimization of (1) gives a unique source vector $s$.

\section{Phase Synchronization}

Definition 1: If $\alpha$ and $\beta$ are two distinct phases, then they are synchronous if and only if $m \alpha-n \beta=C$, where $C$ is a fixed number such that $0 \leq C<\pi$, where $m$ and $n$ are integers [29], [30].

For convenience of calculation, in this paper, we shall keep $m=n=1$. The general case has been dealt with in [31]. Here, we are concerned about processing of stationary EEG signals. So, we can use an FFT-based phase synchronization measuring algorithm. A wavelet-based algorithm for phase synchronization detection, particularly for the nonstationary brain signals, can be found in [32].

Let $x_{j}(t)$ and $x_{k}(t)$ be the EEG signals collected from the $j$ th and $k$ th electrodes, respectively. Their Fourier expansions can be written as follows:

$$
\begin{aligned}
x_{j}(t) & =\frac{a_{j 0}}{2}+\sum_{n=1}^{\infty}\left(a_{j n}^{2}+b_{j n}^{2}\right)^{1 / 2} \sin \left(\frac{2 \pi n t}{p}+\alpha_{j n}\right) \\
\alpha_{j n} & =\tan ^{-1}\left(\frac{a_{j n}}{b_{j n}}\right) \\
x_{k}(t) & =\frac{a_{k 0}}{2}+\sum_{n=1}^{\infty}\left(a_{k n}^{2}+b_{k n}^{2}\right)^{1 / 2} \sin \left(\frac{2 \pi n t}{p}+\alpha_{k n}\right) \\
\alpha_{k n} & =\tan ^{-1}\left(\frac{a_{k n}}{b_{k n}}\right)
\end{aligned}
$$

where $a_{j n}$ and $b_{k n}$ are to be determined by FFT within a suitably chosen time window [33]. If $x_{j}(t)$ and $x_{k}(t)$ are synchronous signals, then the phase lag should be almost uniform across all the harmonics, which is our basic assumption (for mathematical as well as physical motivations, see [33]). We are interested to study not exactly phase-synchronous signals, but almost phasesynchronous signals. In other words, we are looking for

$$
\alpha_{j 1}-\alpha_{k 1} \approx \cdots \approx \alpha_{j n}-\alpha_{k n} \approx \cdots
$$

which implies

$$
\frac{a_{j 1} b_{k 1}-a_{k 1} b_{j 1}}{a_{j 1} a_{k 1}+b_{j 1} b_{k 1}} \approx \ldots \approx \frac{a_{j n} b_{k n}-a_{k n} b_{j n}}{a_{j n} a_{k n}+b_{j n} b_{k n}} \approx \ldots .
$$

This implies that both the mean and the standard deviation of the quantities

$$
\frac{a_{j n} b_{k n}-a_{k n} b_{j n}}{a_{j n} a_{k n}+b_{j n} b_{k n}}-\frac{a_{j n+1} b_{k n+1}-a_{k n+1} b_{j n+1}}{a_{j n+1} a_{k n+1}+b_{j n+1} b_{k n+1}}
$$


over all $n$ would be small when $x_{j}(t)$ and $x_{k}(t)$ are almost phase-synchronous. Let

$$
E(n)=\left|\frac{a_{j n} b_{k n}-a_{k n} b_{j n}}{a_{j n} a_{k n}+b_{j n} b_{k n}}-\frac{a_{j n+1} b_{k n+1}-a_{k n+1} b_{j n+1}}{a_{j n+1} a_{k n+1}+b_{j n+1} b_{k n+1}}\right| .
$$

Since we are interested in phase synchronization only, we have taken $E(n)$ for all $n$ irrespective of the power associated with the band $n$ (for a justification, see [33]). The measure of phase synchronization between $x_{j}(t)$ and $x_{k}(t)$ is given by the syn function defined as

$\operatorname{syn}\left(x_{j}(t), x_{k}(t)\right)=\operatorname{syn}(j, k)=\frac{1}{1+\operatorname{mean}(E(n))+\operatorname{std}(E(n)}$

which gives a measure of synchronization between $x_{j}(t)$ and $x_{k}(t)$ in a 0 (no synchronization) to 1 (perfect synchronization) scale. A complete derivation of the $\operatorname{syn}\left(x_{j}(t), x_{k}(t)\right)$ in pseudocode form has been given in [33]. Since measurements over all $n$ have been incorporated in (9), it actually gives a more minute measure of phase synchronization based on finer resolution than the statistical-correlation-based measures. It can be shown that the time complexity of the algorithm equals to that of the FFT [33].

We have validated this algorithm by simulation on ten artificially generated signals. Each of the signals has been generated by adding a few Fourier components with arbitrary frequency and amplitude. The only exceptions are the sixth and seventh signals as they have been created to be phase-synchronous with each other, in the sense that the seventh has been created from the sixth by uniformly suppressing its amplitude by a constant factor (see [33] for more detail). All other signals are pairwise phase-asynchronous. Out of the ${ }^{10} C_{2}=45$ values of synchronization, 44 values are less than or equal to 0.0881 , and that between the sixth and seventh signal is 1 in a 0 to 1 scale [calculated by (9)]. Another validation comes from the simulation results on a real head model (details presented in Section III). When only a single cortical source is active, it always generates perfectly phase-synchronous signals [with value 1 as calculated by (9)] between any two pairs of scalp EEG electrodes, which is perfectly reasonable.

\section{Clustering}

Here, clustering means the neighborhood construction for each EEG electrode. It should not be confused with clustering in pattern recognition. Fig. 1 gives the position of all the 60 channels on the scalp diagrammatically. By neighborhood of a channel we mean the channel itself along with the channels closest to it (see the examples in Fig. 1). For 60 channels, there are 60 neighborhoods or clusters. The $i$ th cluster, $i \in\{1, \ldots, 60\}$, has been formed according to the following rules.

1) Include the $i$ th channel.

2) Draw a circle centering the $i$ th channel so that at least one channel falls on the circumference and no channel is inside other than the $i$ th channel.

3) Include all the channels falling within the circumference.

It is clear from Fig. 1 that a cluster will contain at least two and at most five channels.

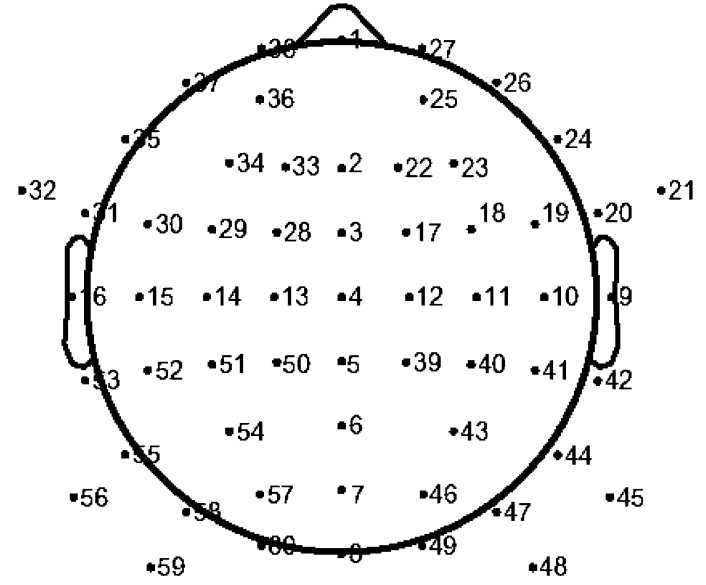

Fig. 1. Locations of all the 60 channels. Formation of two typical clusters according to the nearest channels norm has been shown. Cluster 4 consists of channels $13,14,15,29$, and 51 . Cluster 56 contains only channels 55 and 56.

Calculation of the average cumulative phase synchronization and the average cumulative signal power of the $i$ th cluster have been done according to the following equations:

$$
\begin{aligned}
P(i) & =\frac{1}{|\Lambda|} \sum_{j \in \Lambda} \operatorname{syn}(i, j) \\
W(i) & =\frac{1}{|\Lambda|+1}\left(\sum_{j \in \Lambda} \operatorname{pow}(j)+\operatorname{pow}(i)\right) .
\end{aligned}
$$

Here, $P(i)$ is the average cumulative phase synchronization in the $i$ th cluster, $\Lambda$ is the set consisting of all the channels of the $i$ th cluster other than the $i$ th channel, $|\Lambda|$ is the cardinality of $\Lambda, W(i)$ is the average cumulative signal power of the $i$ th cluster, and $\operatorname{pow}(j)$ denotes the signal power at channel $j$. Since different clusters consist of different numbers of channels, we have taken the average of cumulative values of both the phase synchronization and power of each cluster in order to nullify the effects of channel population in a cluster. The signal power pow $(j)$ can be calculated in many ways. Here, we have taken the mean across all the windows in the Welch method in MATLAB. In this paper, we will often use the words channel and cluster interchangeably.

\section{Simulation OF Source LocAlizATION}

First we have tested our method by simulation on the real head model of the subject constructed from his structural MRI data. The cortex of the subject has been modeled as a grid of triangular mesh consisting of 8959 points. Each point of this cortical mesh is called a cortical point in this paper. Cortex and cortical mesh will be used interchangeably. Precise location of each point has been given in a 3-D Euclidean reference frame. Precise location of each of the 60 scalp electrodes (recorded by MRI) has also been given with respect to the same reference frame. We have constructed each cortical source as a collection of four to seven closely spaced cortical points. They have been activated by artificial time-varying signals, each of which has been generated as a summation of a few arbitrary Fourier 
TABLE I

RESUlTS OF SiMULATION: COMPARISON OF SOURCE LOCALIZATION BY: 1) MN AND 2) SyNCH AND POWER ON A REAL HEAD MOdEL

\begin{tabular}{|l|l|l|}
\hline $\begin{array}{l}\text { Number of } \\
\text { sources } \\
\text { identified } \\
\text { by MN }\end{array}$ & $\begin{array}{l}\text { Number of } \\
\text { sources } \\
\text { identified by } \\
\text { synch and } \\
\text { power }\end{array}$ & $\begin{array}{l}\text { Number } \\
\text { of cases } \\
\text { they both } \\
\text { matched }\end{array}$ \\
\hline 24 & 25 & 23 \\
\hline
\end{tabular}

components (like in Section II-C). These time-varying signals have the same number of time points as the original EEG data collected from the subject during the stimulation. All the cortical points belonging to a single source have been activated by the same signal. The signal represents the event related potential (ERP) generated at the point. Different sources have been activated by different signals. No two sources have been activated by mutually phase-synchronous signals. All nonsource points in the cortical mesh have been assigned zero value uniformly across the time. After activation of the sources, the EEG signals have been generated by the forward model [27], [28] and collected through the 60 scalp electrodes. Then the sources have been localized by: 1) the MN inverse method [26], [28] and 2) the phase synchronization and signal power profile, applied on the collected EEG. In 2), the locations of sources are directly underneath the scalp channels. For comparing 1) and 2), the distance between the sources localized by 1) and 2) has been calculated on the scalp between the channel right on top of the source calculated by 1) and the channel on top of the source calculated by 2). The result has been accepted when both the sources fell within $4.5 \mathrm{~cm}$ of each other as measured on the scalp $(4.5 \mathrm{~cm}$ is enforced by the average interchannel gap in the scalp electrode placements that can be improved by increasing the number of channels). Measurements on the scalp (rather than on the cortex) have been preferred, for it helps us to understand the reliability of method 2) that can only localize a source as a cortical activity under a scalp electrode within a few centimeters of the error range. The results have been summarized in Table I.

When there is only one source, the generated EEG signals collected from any two channels are phase-synchronous $(\operatorname{syn}(i, j)=1$ for all $i, j)$. In the cluster right on top of the source and also in the neighboring clusters, signal power is very high. In all other locations, signal power is very low. In these cases, localizing the source both by MN inverse and by synchronization and signal power profile becomes quite straightforward. So, we have tested our method on scalp EEG signals generated by two cortical sources in each trial: one is stronger than the other by a factor of about 10 . The sources are separated by a distance of $7 \mathrm{~cm}$ or more in the cortex. The sources are either in the somatosensory area or in some neighboring region. Simulated results on 20 trials have been presented in Table I.

Table I shows that for almost $58 \%$ of the total sources and $92 \%$ of the detected sources, localization by MN inverse and by phase synchronization and signal power profile match with each other. In our simulations, in each trial, the two sources have an

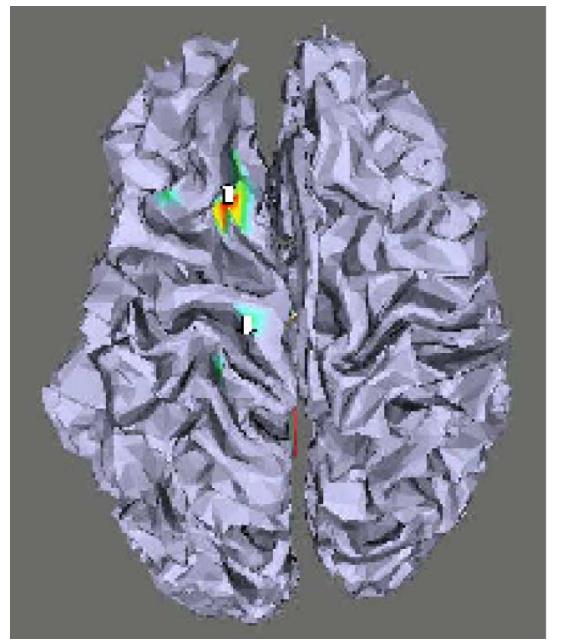

Fig. 2. Cortical source localization during 284th trial of right stimulation $25 \mathrm{~ms}$ after the stimulus onset. The source on somatosensory cortex (below) is rather weak. Arrows indicate possible orientation. The upper source falls right underneath channel 33 and the lower right underneath 13.

intensity disparity of order 10 . The result can be improved by reducing this disparity.

\section{EXPERIMENT AND DATA}

Transcutaneous stimulation of the right median nerve was administered with conventional nicolet bipolar bar electrodes placed $3 \mathrm{~cm}$ apart on a 35-year-old healthy right-handed male volunteer. The stimulation was in the form of constant current square-wave pulses (duration $100 \mu \mathrm{s}, 8 \mathrm{~mA}, 2 \mathrm{~Hz}$ ). During the stimulation scalp, EEG of the subject was collected with SynAmps (Compumedics Neuroscan, El Paso, TX) 64-channel system (data from only 60 channels were used for analysis). Electrodes were placed on the scalp with QuickGel (same company) according to the international 10/10 convention in which channel 14 (Fig. 1) falls right on top of the central position of the left somatosensory cortex. The sample frequency is $5000 \mathrm{~Hz}$, bandpass-filtered between 1 and $1000 \mathrm{~Hz}$. After the bad trial removal, a total of 490 trials have been selected, each of them segmented from $30 \mathrm{~ms}$ before the stimulus onset to $50 \mathrm{~ms}$ afterward.

\section{Results on Human EEG}

To elaborate the derivation of the results on human EEG, analysis of signals from a trial, namely the 284th trial, at 25th $\mathrm{ms}$ after the stimulus onset will be explained. Sources have been localized by $\mathrm{MN}$, supported by high average cumulative phase synchronization and signal power at a nearby (within $4.5 \mathrm{~cm}$, all distances are Euclidean) channel (Fig. 2). Fig. 2 contains only those sources identified both by the MN inverse [1) implemented by OPENMEEG [28]], and phase synchronization and signal power profile across the scalp channels. Identification of sources by synchronization and power profile has been done according to Fig. 3 and Table II. During the stimulation trials, activation of other parts of the cortex beyond somatosensory areas are quite common [34]. This is also shown in Fig. 2. 

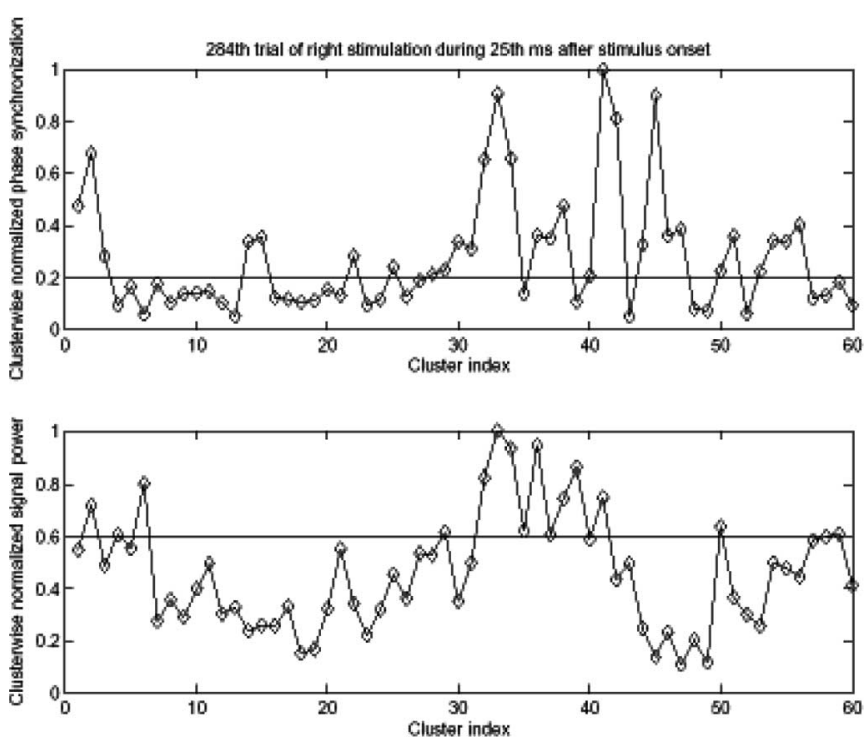

Fig. 3. Mean cumulative phase synchronization and signal power at each cluster during the 284th trial of right stimulation $25 \mathrm{~ms}$ after the stimulus onset. Horizontal lines show thresholding.

TABLE II

ACTIVE CORTICAL POINTS IDENTIFIED BY MN INVERSE DURING THE 284TH Trial Along With Phase Synchronization And Signal Power Profile ON THE SCALP

\begin{tabular}{|l|l|l|}
\hline $\begin{array}{l}\text { Channel } \\
\text { number }\end{array}$ & $\begin{array}{l}\text { \# active cortical } \\
\text { points by MN } \\
\text { within } 4 \mathrm{~cm}\end{array}$ & $\begin{array}{l}\text { Channel numbers with } \\
\text { above threshold values } \\
\text { within } 4.5 \mathrm{~cm}\end{array}$ \\
\hline 13 & 2 & $50(4.15 \mathrm{~cm})$ \\
\hline 28 & 9 & $33(4.32 \mathrm{~cm})$ \\
\hline 33 & 2 & $\begin{array}{l}33(0 \mathrm{~cm}), 34(2.84 \\
\text { cm })\end{array}$ \\
\hline 47 & 2 & None \\
\hline 48 & 2 & None \\
\hline 49 & 2 & None \\
\hline
\end{tabular}

The normalized thresholding for phase synchronization is 0.2 (for the simulated data, it was taken to be 0.4 because the signals were pure). Table II shows the relationship between cortical activities identified by MN method (with half (maximum value) thresholding) and the channel-wise phase synchronization and signal power profile of the scalp EEG signals during the 284th trial of right stimulation $25 \mathrm{~ms}$ after the stimulus onset. Regions with less than two active cortical points have been ignored. Even with half maximum value thresholding, the number of active cortical points remained less than $0.004 \%$ for all 30 trials we have examined. For the MN inverse calculation at the 25 th $\mathrm{ms}$, the average value of the reconstructed sources has been taken from 24.5 to $25.5 \mathrm{~ms}$ in order to have a better SNR. For phase synchronization and signal power profile calculation, the window has been extended by $5 \mathrm{~ms}$ on either side of [24.5 25.5] in order to have a better approximation by the FFT (55 time points).

In some trials, by combining the MN inverse and the phase synchronization and signal power profile, it is even possible to estimate the orientation of the sources. From Table II, it is clear that for the 284th trial, the cortical region below channel 28 (compare Figs. 1 and 2) is the most active, but the high signal power and phase synchronization occur at channel 33 . This indicates that the orientation of the cortical source below channel 28 might be toward channel 33, i.e., right upward (see Fig. 1). Similarly for the cortical source below channel 13, the high signal power and phase synchronization are observed at channel 50, and the orientation of the source is likely to be straight downward.

The phase synchronization and signal power profile of the scalp EEG channels may give a very crude estimation about where a dominant cortical source is likely to be. MN inverse, on the other hand, is a more accurate method. This is the reason why we have ignored the false positives shown in the graphs of Fig. 3. In other words, the synchronization and power profile have been used to further constrain the $\mathrm{MN}$ method in order to better converge toward the actual cortical sources. For this reason, we have ignored the cortical regions shown to be active by $\mathrm{MN}$ below channels 47-49 (Table II). Channel 14 (C3) is situated right on top of the central position of the left somatosensory cortex [24].

It is clear that when two signals are dominated by the same noise, they may appear phase-synchronous to a good extent by (9). In other words, some times phase-asynchronous signals with low SNR may look phase-synchronous. This is clearly not the case here, for when we slid the effective window by just $1 \mathrm{~ms}$, significant changes were observed in the phase synchronization profile while the signal power profile usually remained the same. To emphasize this point, further simulation studies in Section III have been done without any additive noise. In case of the scalp EEG, we have selected trials based on signal amplitude in channels $13,14,15,29$, and 51 that cover the central part of the left somatosensory cortex (Fig. 1). The aforementioned channels are situated at a location where the effects of eye blink and muscle contraction artifacts would be minimal. Moreover, a careful visual inspection of the EEG data in the selected trials did not reveal any abnormal behavior in the aforementioned channels.

We have selected the 25th ms time epoch after stimulus onset by the EEGLAB [23], and the single trials in that epoch according to signal amplitude by measuring the signals across channels 13, 14, 15, 29, and 51 (i.e., cluster 14, Fig. 1) during that time epoch. Selection of trials this way may be thought of a kind of "informed prior" [9], [10]. This makes possible better observation of the effect of stimulus in conformity with known activations. The results of the best 30 trials have been summarized in Table III.

Table III shows the relation between cortical activity and phase synchronization, and signal power becomes weaker with trials consisting of weaker signals in cluster 14. This indicates that the increased synchronization is due to the presence of ERP source rather than noise. Fig. 4 shows the ERP map during all the 490 trials at the 25 th ms after stimulus onset. It also supports the activity profile generated by the MN method during the best 30 trials (Table IV), and the phase synchronization and signal power profile of the scalp channels during that time, as shown in Table IV. Fig. 5 presents a comparison between the 
TABLE III

SUMMARY OF RESULTS FOR THE BEST 30 TRIALS

\begin{tabular}{|l|l|l|l|}
\hline Trial range & $\begin{array}{l}\text { \# channels } \\
\text { identified } \\
\text { with active } \\
\text { cortical } \\
\text { sources (by } \\
\text { MN) } \\
\text { underneath }\end{array}$ & $\begin{array}{l}\text { \# active } \\
\text { cortical } \\
\text { points } \\
\text { identified by } \\
\text { MN }\end{array}$ & $\begin{array}{l}\text { \# channels } \\
\text { with high } \\
\text { synch. and } \\
\text { power within } \\
4.5 \mathrm{~cm}\end{array}$ \\
\hline $\begin{array}{l}\text { Best } 1 \text { to } 10 \\
\text { trials }\end{array}$ & 58 & 185 & $44(75.86 \%)$ \\
\hline $\begin{array}{l}\text { Best } 11 \text { to } 20 \\
\text { trials }\end{array}$ & 72 & 233 & $44(61.11 \%)$ \\
\hline $\begin{array}{l}\text { Best } 21 \text { to } 30 \\
\text { trials }\end{array}$ & 64 & 217 & $38(59.38 \%)$ \\
\hline
\end{tabular}

There are 8959 cortical points in all. A cortial source consists of a few closely spaced cortial points
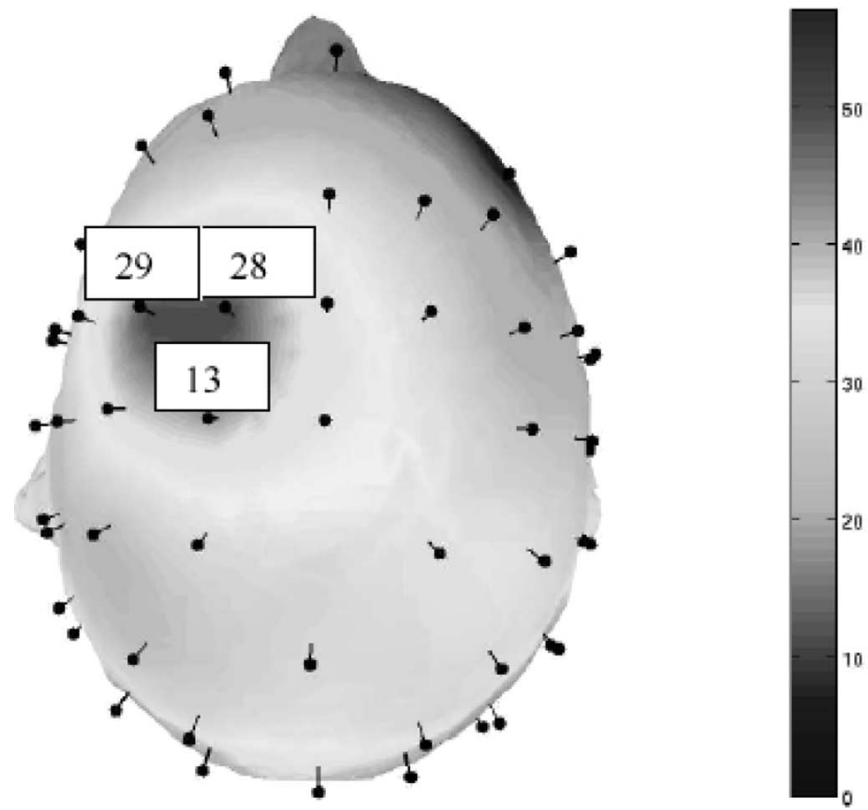

Fig. 4. ERP map of all the 490 stimulation trials by EEGLAB during 25 th $\mathrm{ms}$ after the stimulus onset. The dark spot indicates the most active cortical source of ERP. Channels 13, 28, and 29 are the EEG electrodes underneath which cortical sources have most consistently been identified by MN, and phase synchronization and signal power.

TABLE IV

Profile of Channels 13, 28, AND 29 With the Most ACtive ERP AT 25TH MS DURING THE BEST 30 TRIALS

\begin{tabular}{|l|l|l|l|}
\hline $\begin{array}{l}\text { Chan } \\
\text { no. }\end{array}$ & $\begin{array}{l}\text { \# occurrences } \\
\text { in } 30 \text { trials }\end{array}$ & $\begin{array}{l}\text { \# active cortical } \\
\text { points identified } \\
\text { by MN }\end{array}$ & $\begin{array}{l}\text { \# times } \\
\text { supported by } \\
\text { high synch. } \\
\text { and power }\end{array}$ \\
\hline 13 & 11 & $\begin{array}{l}27(2 \text { points } \\
\text { repeating } 9 \\
\text { times })\end{array}$ & 11 \\
\hline 28 & 15 & $\begin{array}{l}110(8 \text { points } \\
\text { repeating } 11 \\
\text { times). }\end{array}$ & 13 \\
\hline 29 & 11 & 33 & 11 \\
\hline
\end{tabular}

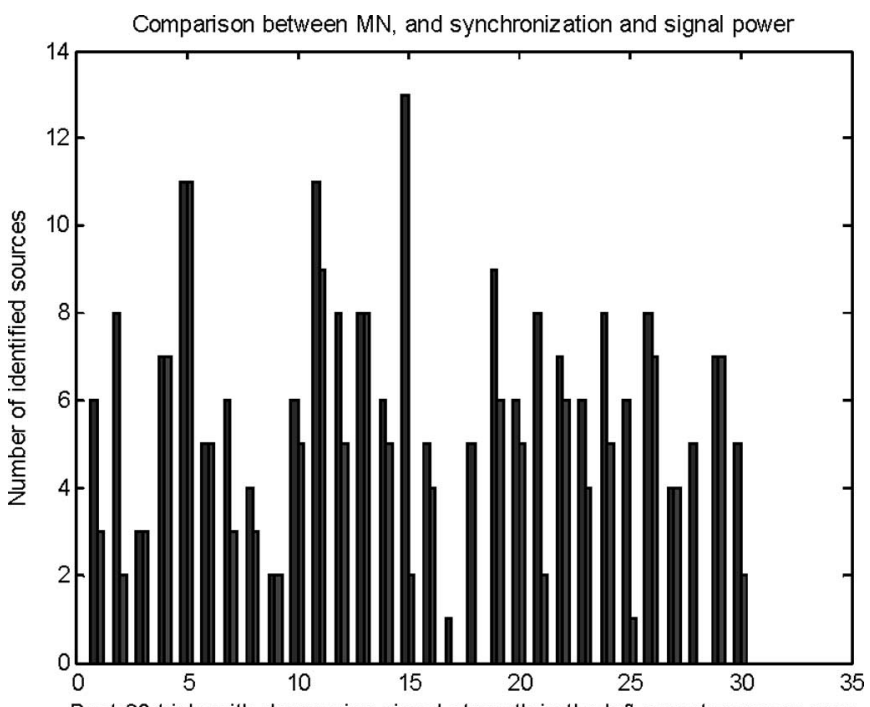

Best 30 trials with decreasing signal strength in the left somatosensory area

Fig. 5. Localized sources in each of the best 30 stimulation trials out of 490 by MN (blue or left bar). Each right bar (red) indicates the subset of the identified sources by MN that have also been identified by phase synchronization and signal power profile of the scalp EEG channels, both $25 \mathrm{~ms}$ after the stimulus onset. Agreements occur most consistently in and around the left somatosensory area (channels 13, 28, and 29).

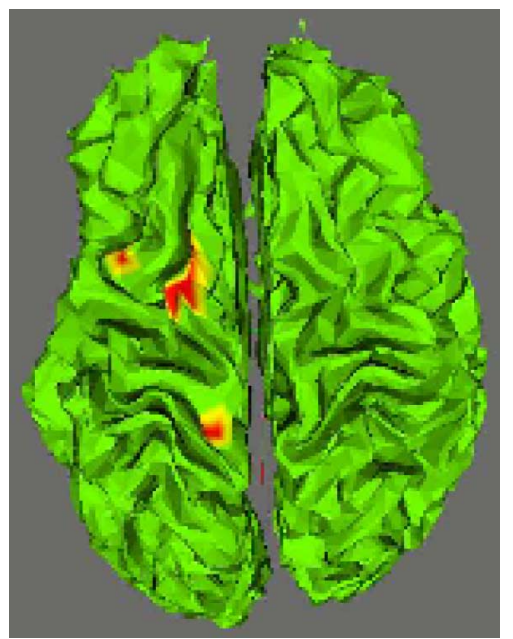

Fig. 6. Dominant cortical sources during $30 \%$ or more of the best 30 trials Sources have been localized in each trial by $\mathrm{MN}$, and phase synchronization and signal power. Cortical points that were active in the largest number of trials (9-11 out of 30) have been taken to reconstruct the sources. Please see the color figure online.

two methods for 30 best trials. It also shows how the sources identified by MN are further refined by synchronization and power profile. Since the agreements between the two methods occur most consistently in and around the left somatosensory area (channels 13, 28, and 29) $25 \mathrm{~ms}$ after the stimulus onset, their combination does make good sense.

It is clear from Table IV that the cortical activity is rather stable underneath channels 13 and 28. Under the channel, 29 active points occurred rather haphazardly. In most cases, active points under channels 13 and 28 occur in the same trial. Active points under channel 29 occur without much relationship 
with the occurrence of active points under channels 13 and 28 . Fig. 6 shows the dominant cortical sources during the 30 best trials, which is consistent with the ERP diagram of Fig. 4. The sources have been localized by MN inverse and also supported by high phase synchronization and signal power on the scalp. Orientations are different in different trials, and therefore, have not been shown.

\section{CONCLUSION}

In this paper, we have applied the classical MN inverse solution for the scalp EEG source localization during median nerve stimulations simultaneously, but independently, with the phase synchronization and power profile of the EEG signals in single trials. Both the methods agree with each other most consistently underneath channels 13, 28, and 29 (covering parts of somatosensory and adjacent areas), where the ERP is the strongest at 25 th ms after the stimulus onset. This indicates a more accurate (certain) single-trial source localization by combining the two methods rather than by just one.

Here, we have described an FFT-based phase synchronization measuring algorithm whose time complexity is equal to that of the FFT. It may be useful for processing epileptic EEG signals [18], particularly for seizure focus lateralization [30]. The algorithm can capture interesting variations in synchronization during sliding the window even by as small a time step as $1 \mathrm{~ms}$ while the signal power profile remains unchanged, as has been observed on human scalp EEG data. This indicates that the synchronization measure may not be due to noise. An interesting future study would be to see how it performs with varying SNR. However, during the present study, it has been able to localize sources in single trials of noise-free simulated EEG as well as human scalp EEG (bandpass-filtered between 1 and $1000 \mathrm{~Hz}$ ) with comparable efficiency with $\mathrm{MN}$ when the signal power is high.

In this paper, we have been constrained by the interchannel gaps in the EEG net, which, in most cases, are more than $3 \mathrm{~cm}$, and in some cases, more than $4 \mathrm{~cm}$. So we could not improve the results presented in this paper, where the resolution of distance could not be shortened less than $4.5 \mathrm{~cm}$. The results are likely to be better with a denser EEG net.

A future goal will be to estimate the priors with phase synchronization and signal power profile across the scalp EEG channels and then apply the estimation maximization in a Bayesian framework in order to more accurately estimate the sources with the help of the weighted MN algorithm, as described in [8]-[10].

\section{ACKNOWLEDGMENT}

The author would like to thank M. Clerc, T. Papadopoulo, O. Faugeras, and E. Tognoli for many lively discussions and critical evaluations. He would also like to thank P. Marquis for data acquisition and preprocessing.

\section{REFERENCES}

[1] Z. J. Koles, "Trends in EEG source localization," Eletroencephalogr. Clin. Neurophysiol., vol. 106, pp. 127-137, Feb. 1998.
[2] S. Debener, M. Ullsperger, M. Siegel, and A. K. Engel, "Single-trial EEG-fMRI reveals the dynamics of cognitive function," Trends Cogn. Sci., vol. 10, pp. 558-563, Dec. 2006.

[3] P. L. Nunez and R. B. Silberstein, "On relationship of synaptic activity to macroscopic measurements: Does co-registration of EEG with fMRI make sense?," Brain Topogr, vol. 13, pp. 79-96, 2000.

[4] S. Baillet, J. C. Mosher, and R. M. Leahy, "Electromagnetic brain mapping," IEEE Signal Process. Mag., vol. 18, no. 6, pp. 14-30, Nov. 2001.

[5] B. N. Cuffin, "EEG localization accuracy improvements using realistically shaped head models," IEEE Trans. Biomed. Eng., vol. 43, no. 3, pp. 299303, Mar. 1996.

[6] A. Rodriguez-Rivera, B. V. Boryshnikov, B. D. V. Veen, and R. T. Wakai, "MEG and EEG source localization in beamspace," IEEE Trans. Biomed. Eng., vol. 53, no. 3, pp. 430-441, Mar. 2006.

[7] L. Parra, C. Alvino, A. Tang, B. Pearlmutter, N. Yeung, A. Osman, and P. Sajda, "Single-trial detection in EEG and MEG: Keeping it linear," Neurcomputing, vol. 52-54, pp. 177-183, 2003.

[8] K. J. Friston, W. Penny, C. Phillips, S. Kiebel, G. E. Hinton, and J. Ashburner, "Classical and Bayesian inference in neuroimaging: Theory," NeuroImage, vol. 16, pp. 465-483, 2002.

[9] C. Phillips, J. Mattout, M. D. Rugg, P. Maquet, and K. J. Friston, "An empirical Bayesian solution to the source reconstruction problem in EEG," NeuroImage, vol. 24, pp. 997-1011, 2005.

[10] J. Mattout, C. Phillips, W. D. Penny, M. D. Rugg, and K. J. Friston, "MEG source localization under multiple constrains: An extended Bayesian framework," Neurolmage, vol. 30, no. 3, pp. 753-767, Apr. 2005.

[11] C. von der Malsburg and W. Schneider, "A neural cocktail-party processor," Biol. Cybern., vol. 54, no. 1, pp. 29-40, May 1986.

[12] C. Tallon-Baudry, O. Bertrand, C. Delpuech, and J. Pernier, "Oscillatory $\gamma$-band $(30-70 \mathrm{~Hz})$ activity induced by a visual task in humans," $J$. Neurosci., vol. 17, pp. 722-734, 1997.

[13] J. P. Lachaux, E. Rodriguez, J. Martinerie, and F. J. Varela, "Measuring phase synchrony in human brain signals," Hum. Brain Mapp., vol. 8, pp. 194-208, 1999.

[14] E. Rodriguez, N. George, J. P. Lachaux, J. Martinerie, B. Renault, and F. J. Varela, "Perception's shadow: Long-distance synchronization of human brain activity," Nature, vol. 397, pp. 430-433, 1999.

[15] A. K. Engel, P. Fries, and W. Singer, "Dynamic predictions: Oscillations and synchrony in top-down processing," Nat. Rev. Neurosci., vol. 2, pp. 704-716, 2002.

[16] C. Brunner, R. Scherer, B. Graimann, G. Supp, and G. Pfurtscheller, "Online control of a brain-computer interface using phase synchronization," IEEE Trans. Biomed. Eng., vol. 53, no. 12, pp. 2501-2506, Dec. 2006.

[17] M. Chavez, M. L. van Quyen, V. Navarro, M. Baulac, and J. Martinerie, "Spatio-temporal dynamics prior to neocortical seizures: Amplitude versus phase couplings," IEEE Trans. Biomed. Eng., vol. 50, no. 5, pp. 571583, May 2003.

[18] F. Mormann, T. Kreuz, R. G. Andrzejak, P. David, K. Lehnertz, and C. E. Elger, "Epileptic seizures are preceded by a decrease in synchronization," Epilepsy Res., vol. 53, pp. 173-185, 2003.

[19] C. Hammond, H. Bergman, and P. Brown, "Pathological synchronization in Parkinson's disease: Networks, models and treatments," Trends. Neurosci., vol. 30, no. 7, pp. 357-364, May 2007.

[20] L. D. Iasemidis, "Epileptic seizure prediction and control," IEEE Trans. Biomed. Eng., vol. 50, no. 5, pp. 549-558, May 2003.

[21] F. Mormann, K. Lehnertz, P. David, and C. E. Elger, "Mean phase coherence as a measure of phase synchronization and its application to the EEG of epilepsy patients," Physica D, vol. 53, pp. 358-369, 2000.

[22] R. Q. Quiroga, A. Kraskov, T. Kreuz, and P. Grassberger, "Performance of different synchronization measures in real data: A case study on electroencephalographic signals," Phys. Rev. E, vol. 65, pp. 041903-1-041903-14, 2002.

[23] EEGLAB - Open source MATLAB tool box for eletrophysiological research. (2007). [Online]. Available: sccn.ucsd.edu/eeglab/.

[24] K. Inui, X. Wang, Y. Tamura, Y. Kaneoke, and R. Kakigi, "Serial processing in human somatosensory system," Cereb. Cortex, vol. 14, no. 8, pp. 851-857, Aug. 2004.

[25] N. Forss, R. Hari, R. Salmelin, A. Ahonen, M. Hamalainen, M. Kajola, and J. Simola, "Activation of the human posterior parietal cortex by median nerve stimulation," Exp. Brain Res., vol. 99, no. 2, pp. 309-315, May 1994.

[26] G. Adde, M. Clerc, and R. Keriven, "Imaging methods for MEG/EEG inverse problem," Int. J. Bioelectromagn. (IJBEM), vol. 7, no. 2, pp. 111114, 2005. 
[27] J. Kybic, M. Clerc, T. Abboud, O. Faugeras, R. Keriven, and T. Papadopoulo, "A common formalism for the integral formulations of the forward EEG problem," IEEE Trans. Med. Imag., vol. 24, no. 1, pp. 1218, Jan. 2005.

[28] OPENMEEG (2007). [Online]. Available: http://lists.gforge.inria.fr/ pipermail/openmeeg-commits/2007-May/000088.html

[29] M. Rosenblum, A. Pikovsky, J. Kurths, C. Schafer, and P. A. Tass, "Phase synchronization: From theory to data analysis," in Neuro-Informatics and Neural Modeling, F. Moss and S. Gielen, Eds. Amsterdam, The Netherlands: Elsevier, 2001, pp. 279-321.

[30] M. Caparos, V. Louis-Dorr, F. Wendling, L. Millard, and D. Wolf, "Automatic lateralization of temporal lobe epilepsy based on scalp EEG," Clin. Neurophysol., vol. 117, pp. 2414-2423, 2005.

[31] P. Tass, M. G. Rosenblum, J. Weule, J. Kurths, A. Pikovsky, J. Volkmann, A. Schnitzler, and H.-J. Freund, "Detection of $n: m$ phase locking from noisy data: Application to magnetoencephalography," Phys. Rev. Lett., vol. 81, pp. 3291-3294, 1998.

[32] J. P. Lachaux, A. Lutz, D. Rudrauf, D. Cosmelli, M. L. Van Quyen, J. Martinerie, and F. J. Varela, "Estimating the time-course of coherence between single-trial brain signals: An introduction to wavelet coherence," Neurophysiol. Clin., vol. 32, pp. 157-174, 2002.

[33] K. Majumdar. (2008, Apr.). An FFT based measure of phase synchronization [Online]. Available: http://arxiv.org/ftp/q-bio/papers/ 0612/0612004.pdf

[34] J. Ruben, J. Schwiemann, M. Docuchert, R. Meyer, T. Krause, G. Curio, K. Villringer, R. Kurth, and A. Villringer, "Somatotopic organization of human secondary somatosensory cortex," Cereb. Cortex, vol. 11, no. 5, pp. 463-473, May 2001.

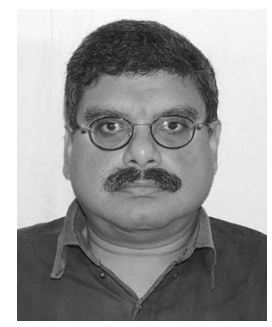

Kaushik Majumdar (M'06) received the B.Sc. degree from the University of Calcutta, Kolkata, India, in 1990, the M.Sc. degree in mathematics from Annamalai University, Annamalai Nagar, India, in 1996, and the M.Tech. and Ph.D. degrees in computer science from the Indian Statistical Institute, Kolkata, in 1999 and 2003, respectively.

During 2003-2004, he was a Research Associate in the Council of Scientific and Industrial Research, Government of India. During 2005-2006, he was a Fulbright Fellow in the Department of Computer Science, University of Memphis. In 2006, he worked jointly as an R\&D Scientis at Electrical Geodesics, Inc., and as a Research Associate at the University of Oregon, Eugene. From 2006 to 2007, he was a Postdoctoral Fellow at the Institute of Mathematical Sciences, Chennai, India. From July 2007 to August 2008, he was an Invited Scientist in the Odyssee Group, Institut National de Recherche en Informatique et en Automatique (INRIA) Sophia Antipolis, France. He is currently a Postdoctoral Fellow in the Center for Complex Systems and Brain Sciences, Florida Atlantic University, Boca Raton. He has authored or coauthored more than 20 papers published in international journals and conferences. His current research interests include neural signal processing, mathematical and computational modeling of the brain functions, and nonlinear system theoretic studies of epilepsy.

Dr. Majumdar is a member of the American Mathematical Society. He was a recipient of the Norbert Wiener Outstanding Paper Award in 2005 for a paper in Kybernetes in 2004. Since 2007, his name has been featured in Marquis Who's Who of the World. 